\title{
A REVIEW ON IOT BIG DATA: AN INTERCONNECTION BETWEEN THE FUTURE TECHNOLOGIES
}

\author{
Lekha C. Warrier ${ }^{1}$ \\ ${ }^{I}$ Assistant Professor, ISBR COLLEGE, Electronic City, Bangalore
}

\begin{abstract}
In the digital world, society has become data driven, all the decision we are making as data oriented. The amount of data generating in each fraction of second is increasing exponentially. The effect of Internet of Things has made significant importance in the field of big data. Both the technologies like Big Data and Internet of Things can go hand in hand. People cannot spend a day without the support of IOT. The analysis of the tremendous data requires more skilled people to work on it. The effect of IOT has made all the industries to use efficient big data technologies to store, process and analyse the data. This paper reviews the relationship between IOT and Big Data and explaining about the data security challenges.
\end{abstract}

Keywords - Internet of Things, Big Data, Data Security, Real time data, Challenges.

\section{INTRODUCTION}

The role of artificial Intelligence in the near future will be highly remarkable. The substance of machine to machine communication would be ruling the information industry in the coming years. All the devices we are using for our day today life are generating data in a tremendous manner. As the people are digitally literate, the communication, transaction the education everything getting done with digital devices. As a result of this the bunch amount of data are coming out from each machines in a second. We can tell that enhancement of technology is producing a lot of data.

IOT and Big Data are the latest technologies in which IT environment relies. As the magnification of data is enlarging in every fraction of second, the relevance of acquiring meaningful data has become important. Everywhere we are seeing different forms of data. The valuable insight from these data need to be extracted for the decision making purpose in the market.

In this paper we are giving a vivid idea about IOT, the connection of IOT with Big Data and the impact of both in todays. The IOT makes the smart world to use Big Data terminology and technology for better storage and analysis of the data.

\section{INTERNET OF THINGS}

Internet of Things is the most popular word in the information industry. IOT simply connecting all the surrounding smart devices (things) to the internet. These devices use sensors and actuators to communicate with each other across the internet [4]. IOT consists of technologically advanced objects that have the ability to transmit information and potentially even sense, monitor and react to human behaviour. IOT acts as an ecosystem of connected physical objects that are accessible through the internet. With the IoT, the possibilities are endless.
From homes and industries to enterprises, IOT have become a common place in all walks of life. The requirement of IOT came to matter when the people and devices wanted to receive more data and especially things wanted to get automated and faster.

IOT is fuelled by the recent advances of a variety of devices and communication technologies, but things included in IOT are not only complex devices such as mobile phones, but they also comprise everyday objects such as food, clothing, furniture, paper, landmarks, monuments etc. This growing number of smart, connected products can present opportunities to businesses by providing insights that can enhance productivity and increase revenue. The IOT includes ideas such as smart homes and connected appliances, as well as smart cities.

Hard ware devices like sensors and actuators will capture the data from different sources (Pressure, Temperature etc). All the devices are connected to the cloud to make intelligent systems. These systems are connected to the cloud using some protocols like Ethernet or Wi-Fi. The communication or transmission of data happens using some internet protocols like UDP, TCP .The device's data will be stored and managed with optimised time series backend. By sending some notification or giving alert to the user, IOT makes the information relevant [9].

\section{BIG DATA}

Big data is defined as the collection of large and complex data sets that it becomes difficult to process with traditional data processing applications. The challenges accompanied with big data are capture, curation, storage, search, share, transfer, analysis, and visualization of large amount of data [6] 
Big data is enabling organisations to collect and analyse data in new ways, helping to transform businesses, industry, government services and people's lives. It refers not only to the size of the data sets, but also to the speed at which the data is created and analysed, along with the variety of tools or data analytics solutions that can be used.

The quantity of data produced in every second from various sources is beyond the capacity of the existing technologies. To make the relevant data from the pool of data, extraction is required. To store and process the huge amount of data some new techniques had to be introduced. With that purpose Big Data had come to the screen of information industry. The use of internet only made the word big data popular.

As the technology grows faster in every dimensions, the world become smart in every sense. The mobile phone, TV, Refrigerator, Car all have capacity to transmit and communicate via digitally with or without human intervention. The business organisations in public and private sectors completely depend on big data technologies. As the growth and availability of data became huge, the traditional technologies are not enough to process it. Big data enables businesses to make the most of both historic and real-time data that is generated through supply chains, production processes and customer behaviours.

The Big Data can be described with three terms.

Velocity: The rate at which data is flowing can be called as velocity. In a Nano second if millions of data is curving through different mediums, then the meaningful data should be extracted and processed in an efficient manner. Velocity of data also is increasing rapidly over time for both structured and unstructured data, and there's a need for more frequent decision making about that data. For example, the rapidity of social media use is increasing day by day. The extraction of relevant data should be very fast to make it up in an efficient manner.

Volume: The quantity of data from various sources can be called as the volume of data. The more data become, the more space is required to process the data. RFID tags appear on inventory items capturing transaction data as goods are shipped through the supply chain. Big data can also refer to the exploding information available on social media such as Facebook and Twitter.

Variety: The data coming from diverse sources can be defined as variety of the data. The data can be an image file or video or in any other format.

\section{RELATIONSHIP BETWEEN IOT AND BIG}

\section{DATA}

The Combination of IOT and Big Data is an unexplored research area that has brought new and interesting challenges for achieving the goal of future smart world [1].Intelligence is added to everyday things in our current world and the artificial intelligence and machine learning technologies predicts our future. Simply we can define IOT as utilizing the internet as much as computer do. The real value of IOT is about creating smarter products, delivering intelligent insights and providing new business outcomes []. As the millions of devices get connected IOT will trigger massive inflow of big data. To convert the massive bunches of data into actionable events big data technologies should be used efficiently. IOT data analytics will provide better infrastructure for storage and computing. IOT contains only really valuable data that is actionable.

The main focus of every business is customer satisfaction and profit. To understand the feelings of customer, the interaction is very much important. A medium should be there to communicate with consumers. The feedback collecting by companies will help to make their products and services better. Based on the suggestions and demands of people they do the corresponding changes to the product. Data driven decision making is done using big data. For better future decisions the analysis of big data is essential.

As data generated is increasing in every seconds, people are not getting use of it. There should be a way to process the rapid generated different varieties of data. Organisations are generating pool of data. But they are hardly using a less amount of data because using our traditional software it is not possible to process the high volume of data. Now it is evident that big data is too big, too fast and too disparate data which could not be processed by the traditional database architectures and need special sophisticated systems [2]. The mountain of data that Internet of Things produces would be useless without the analytic power of big data. Better decisions will lead the company in all aspects. Moving data into information, that information to insights, those insights to actions is the main strategy of decision making. The IOT is a fast-growing constellation of internetconnected sensors attached to a wide variety of 'things'. Put simply; it is the concept of connecting any device with an on / off switch, to the Internet and to each other, facilitating a large network of connected 'things'. According to analyst firm Gartner, by 2020, there will be over 26 billion connected devices, although predictions vary depending on the source [4].

Internet of Things is directly and indirectly associated with daily lifestyle products across the globe. Internet connectivity is one of the major requirements of different application as it is expected to grow at high rate in next forecast period. [10]

Beyond laptop and mobile the area of connectivity is enlarging. World is becoming smart with smart wearables, smart homes, smart cars etc. Home appliances connected with internet will notify everything in your mobile number like in refrigerator vegetables are over, Air conditioner is not in working condition etc. All these devices emanating millions of data in a day which leads to big data paradigm. 
Table 1: GROWTH OF IOT [10]

\begin{tabular}{|l|l|}
\hline Year & $\begin{array}{l}\text { Number of } \\
\text { Connected Device }\end{array}$ \\
\hline 1990 & 0.3 million \\
\hline 1999 & 90.0 million \\
\hline 2010 & 5.0 billion \\
\hline 2013 & 9.0 billion \\
\hline 2025 & 1.0 trillion \\
\hline
\end{tabular}

\section{IMPACT OF IOT BIG DATA IN OUR DAILY}

\section{LIFE}

In this section some applications of IOT big data is explaining briefly, which we are using for our day to day activities.

1. Smart Home: To make our life more simpler and convenient, IOT big data technology direct us towards a technically sounded ecosystem. Before us entering to our house we could on all the lights and keep track of the pets inside your home. The data generated by the devices collected and analysed will give more information for making some valuable decisions.

2. Connected Cars: The revolution in auto mobile has happened by the announcement of Tesla, the self-driven car. More companies are working on this concept using on-board sensors and inter connectivity. Big data, as produced by this cars are analysed in real time and gives meaningful insights.

3. Smart Wearables: New generation people are more conscious about their health, using IOT enables devices they can track their calories burnt, the pressure, their heart beat etc.

4. Smart Retail: To serve the consumers better, the retail sector utilising IOT in enormous ways. It helps the retailors to remain connected with their customers and gets feedback from them about the products and services.

\section{SECURITY CHALLENGES IN IOT BIG DATA}

Apart from the relevance of IOT in our daily life, the vulnerability creating by the connected things is increasing day by day. IOT, the future of internet will play a vital role in our daily activities. The use of IOT is expected to grow rapidly in near future. The big data tools will analyse and process the plenty of data generated by the IOT applications. The increased usage of connected devices lead IOT networks to a high prone security attacks. Efficient mechanism for the proactive and reactive security in IOT data is very essential to gain the customer confidence.

Privacy is under high threats in this technical world. All the personal information is attached to the smart devices and the administration of this personal data has become very poor. Authentication and authorisation of your personal data before transmitting is very important now a days. End to end encryption of the data can be applied for reducing the security challenge to some extent. Device manufacture will need to upgrade the software in a regular period of time to avoid security breaches. The owner of IOT systems have to make sure that their devices and software system remain up to date.

The big data tools like for processing and storage of this large quantity of data Hadoop and NoSQL can create vulnerabilities for authentication and network. The distributed nature of the IOT big data environment is a complicated challenge in this field. As the diverse data is generating from the various sources, the security breaches is expected at any point of time.

A. IOT Network Security: The security in IOT network is more complex compared to the traditional level security. The fast emerging network belongs to Internet of Things in the current world. Fire wall protection, intrusion techniques are very much important in the data security concern.

B. IOT Authentication: The users will have the credentials to authenticate the IOT devices. Thus the limitation to the access of devices joining the network can be defined. Thus the noisy data which is generated from the unnecessary users can be reduced to some extent.

C. IOT Encryption: By using cryptographic algorithms for securing data between IOT devices and backend systems, the data integrity can be preserved.

D. IOT Security Analytics: IOT security analytics will increasingly be required to detect IOT-specific attacks and intrusions that are not identified by traditional network security solutions such as firewalls. Collecting and aggregating data from the IOT devices, using the security measures adds the flexibility in machine learning, big data technologies and artificial intelligence.

\section{CONCLUSION}

The technically driven society has made significant impact on daily life of human being. The day to day activities of people have been synchronized using smart devices. Life has become smart in all the aspects through IOT technology. The data generated by IOT devices have to be analyzed, in efficient manner. For the sake of this, big data technology had been widely recommended. The data security must be assured for these emerging technologies. In this paper we have studied the concept of IOT, big data and the relationship between them in real world. The security challenges facing by the connected devices at the time of collecting and analyzing using big data also explained in detail.

\section{REFERENCES}

[1] Daniel E. O'Leary,"Artificial Intelligence and Big Data" IEEE Intelligence System ,pp.1541-1672,2013

[2] Zaheer Khan,Ashiq Anjum,Kamran Soomro ,Muhammad Atif Tahir "Towards cloud based big data analytics for smart future cities.",Journal of Cloud Computing : Advances \& Systems ,Springer 2015 
[3] G Gomathi,R Sangeethapriya,'Impact of Big Data in IOT: A Review",IRJAET,pp.1748-1752,March 2017.

[4] K.R Kundhavai, S.Sridevi,'IoT and Big Data- The Current and Future Technologies: A Review", IJCSMC, Vol. 5, Issue. 1, January 2016.

[5] Pratap Kumar, Rakesh Singh Kunwar,Alok Sachan,"A Survey Report on: Security \& Challenges in Internet of Things" in proc National Conference on ICT \& IOT,pp.35-39,Jan 2016.

[6] Min Chin, Shiwin Mao, Yunhao Liu,'Big Data : A Survey", Springer, 171-209, 2014

[7] Munesh Kataria, Pooja Mittal, Big Data: A Review, IJCSMC, Volume 3, Issue-7, pp. 106-110, , July 2014

[8] Nasser Thabet,Tariq Rahimm Soomro,"Big Data Challenges",Journal of Computer Engineering \& Information Technology",September 2015

[9] Lopez Research "Introduction to the Internet of Things",November,2013.

[10] Analytics Vidhya,"10 Real World Applications on Internet of Things", https://www.analyticsvidhya.com/blog/2016/08/10youtube-videos-explaining-the-real-worldapplications-of-internet-of-things-iot/ 